\title{
Caracterización y propiedades mecánicas a alta temperatura de un acero inoxidable dúplex
}

\author{
J.A. Jiménez ${ }^{(*)}$, M. Carsí ${ }^{(*)}$ y O.A. Ruano ${ }^{(*)}$ \\ Resumen Se ha estudiado la microestructura y el comportamiento mecánico a alta temperatura de un acero ino- \\ xidable dúplex procesado termomecánicamente. Durante el calentamiento a la temperatura de ensa- \\ yo, el material recristaliza y se obtiene una microestructura de granos austeníticos de tamaños com- \\ prendidos entre 10 y $15 \mu \mathrm{m}$ agrupados en islas incluidas en una matriz más o menos continua de \\ ferrita. Ensayos de tracción a temperaturas superiores a $1.000^{\circ} \mathrm{C}$ y bajas velocidades de deformación \\ muestran un exponente de la tensión igual a 2 y alargamientos a rotura de hasta $290 \%$. Estos valores \\ permiten asociar el mecanismo de deformación al deslizamiento de fronteras de grano, el cual deter- \\ mina la desaparición progresiva de las islas de granos austeníticos durante la deformación. Finalmen- \\ te, se encontró un valor de $167 \mathrm{~kJ} / \mathrm{mol}$ para la energía de activación de la deformación plástica, la \\ cual se relacionó con la energía de autodifusión del hierro a lo largo de las fronteras de grano.
}

Palabras clave: Aceros dúplex. Fluencia. Propiedades mecánicas.

\section{Characterization and high temperature mechanical properties in a duplex stainless steel}

\begin{abstract}
The microstructure and mechanical behavior at high temperature of a thermomechanical processed duplex stainless steel have been studied. Recrystalization of the material takes place during heating to test temperature, and a microstructure consisting of islands of austenitic grains of about 10-15 $\mu \mathrm{m}$ in size included in a more or less continuous matrix of ferrite is observed. Tensile tests at temperatures above $1,000{ }^{\circ} \mathrm{C}$ and at low strain rates show a stress exponent of about 2 and elongations to failure up to $290 \%$. These values suggest that deformation is controlled by a grain boundary sliding mechanism, which causes a decrease in the size of the islands during deformation. Finally, an activation energy for plastic deformation of $167 \mathrm{~kJ} / \mathrm{mol}$ was observed that was related to the activation energy for grain boundary diffusion of iron.
\end{abstract}

Keywords: Duplex steels. Creep. Mechanical properties.

\section{INTRODUCCIÓN}

Los aceros inoxidables dúplex tienen gran interés para aplicaciones en las que se requiere un material que combine buena resistencia a la oxidación y a la corrosión con buenas propiedades mecánicas $(1$ y 2 ). Las elevadas características de resistencia, ductilidad y tenacidad de estas aleaciones

(*) Centro Nacional de Investigaciones Metalúrgicas, CENIM (CSIC), Avda. de Gregorio del Amo 8, 28040-Madrid (España). están relacionadas con la presencia de una microestructura bifásica compuesta por ferrita $(\delta)$ y austenita $(\gamma)$ : por un lado, la ferrita es responsable de la resistencia y, por otro, la austenita asegura un elevado valor de la tenacidad (3). Para conseguir una determinada fracción de volumen de cada fase en la microestructura es necesario realizar un riguroso control del contenido de los elementos aleantes, sobre todo cromo y níquel. Sin embargo, también se suelen añadir, en menor cantidad, elementos como nitrógeno, cobre, molibdeno, silicio, manganeso y volframio con objeto de equilibrar la estructura y mejorar el comportamiento a la corrosión (4). 
Para evitar la fragilización de estas aleaciones por precipitación de fase $\sigma$ es necesario evitar durante el proceso de manufactura una prolongada exposición a temperaturas comprendidas entre 450 y $900^{\circ} \mathrm{C}(5$ y 6$)$. Una solución a este problema es la conformación superplástica de las aleaciones a temperaturas superiores a $1.000{ }^{\circ} \mathrm{C}$. Para ello, y como paso previo, es necesario conseguir una microestructura de grano fino mediante un tratamiento termomecánico adecuado, que incluye, como etapa final, una deformación en frío del $50 \%$ mediante un proceso de laminación (7 y 8).

En el presente trabajo se ha analizado la microestructura y el comportamiento mecánico de un acero dúplex comercial. Se desea comprobar en qué condiciones el mecanismo que controla la deformación plástica a temperatura elevada puede asociarse a un deslizamiento de fronteras de grano, propio del comportamiento superplástico.

\section{PROCEDIMIENTO EXPERIMENTAL}

Se utilizó un acero dúplex de composición, en \% en masa, $25 \mathrm{Cr}-6 \mathrm{Ni}-3 \mathrm{Mo}$, suministrado en forma de chapa. Dicha chapa fue sometida por el suministrador a un tratamiento termomecánico que incluyó laminación en caliente y en frío de la misma. Para el estudio microestructural de la chapa y de las probetas ensayadas en tracción se utilizaron técnicas de difracción de rayos $\mathrm{X}$, microscopía óptica y electrónica de barrido. Las propiedades mecánicas se determinaron mediante ensayos de tracción con cambios en la velocidad de deformación entre $3 \times 10^{-6}$ y $10^{-2} \mathrm{~s}^{-1}$ a temperaturas comprendidas entre 1.000 y $1.100{ }^{\circ} \mathrm{C}$. Se emplearon probetas planas con una sección transversal de $3 \times 5 \mathrm{~mm}$ y una longitud útil de $20 \mathrm{~mm}$, mecanizadas de modo que la dirección de tracción fuese paralela a la dirección de laminación.

\section{RESULTADOS}

\subsection{Microestructura}

La figura 1 muestra la microestructura del material de partida. Se observa una microestructura gruesa consistente en islas de austenita alargadas en la dirección de laminación incluidas en una matriz más o menos continua de ferrita. Esta microestructura de partida desaparece cuando se somete la chapa a un tratamiento térmico a una temperatura comprendida entre 1.000 y $1.100{ }^{\circ} \mathrm{C}$. Durante este tratamiento, el material recristaliza y se obtiene una microestructura como la presentada en la figura 2 . Dicha figura ilustra cómo, tras un recocido de 90 min a $1.050{ }^{\circ} \mathrm{C}$, se obtiene una microestructura de

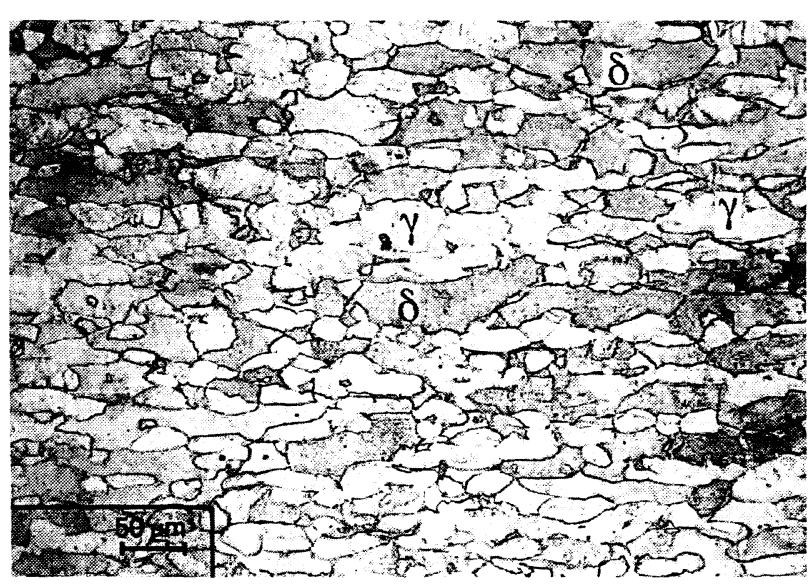

FIG. 1.- Microestructura del material de partida.

FIG. 1.-Microstructure of the as-received material.

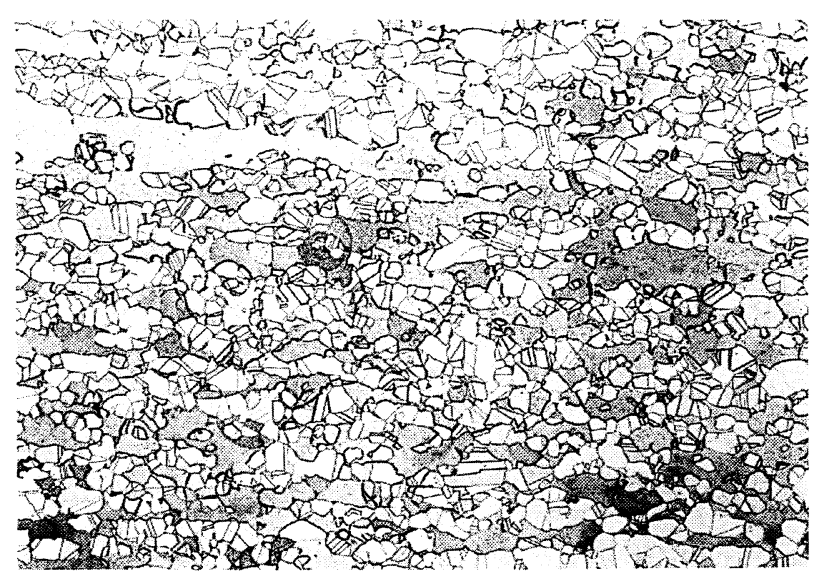

FIG. 2.- Microestructura después de un tratamiento térmico a $1.050^{\circ} \mathrm{C}$ (mismo aumento que Fig. 1).

FIG. 2.- Microstructure after annealing at $1,050^{\circ} \mathrm{C}$ (same magnification as Fig. 1).

granos austeníticos de unos $15 \mu \mathrm{m}$ de tamaño, agrupados en colonias. Dichos granos austeníticos presentan frecuentemente maclas en su interior.

\subsection{Comportamiento mecánico}

El comportamiento mecánico en el intervalo de temperaturas comprendido entre 1.000 y $1.100{ }^{\circ} \mathrm{C}$ puede observarse en las curvas log-log de velocidad de deformación real, $\dot{\varepsilon}$, en función de la tensión real corregida por el módulo de Young, $\sigma / E$, que se presentan en la figura 3. Como puede apreciarse, se produce una disminución del exponente de la tensión desde 5 hasta un valor próximo a 2 a medida que se disminuye la velocidad de deformación. A pesar de que, en función de las condiciones de ensayo, el material presenta dos regímenes bien diferenciados, la energía de activación medida en ambos es muy similar, alrededor de $170 \mathrm{~kJ} / \mathrm{mol}$. 


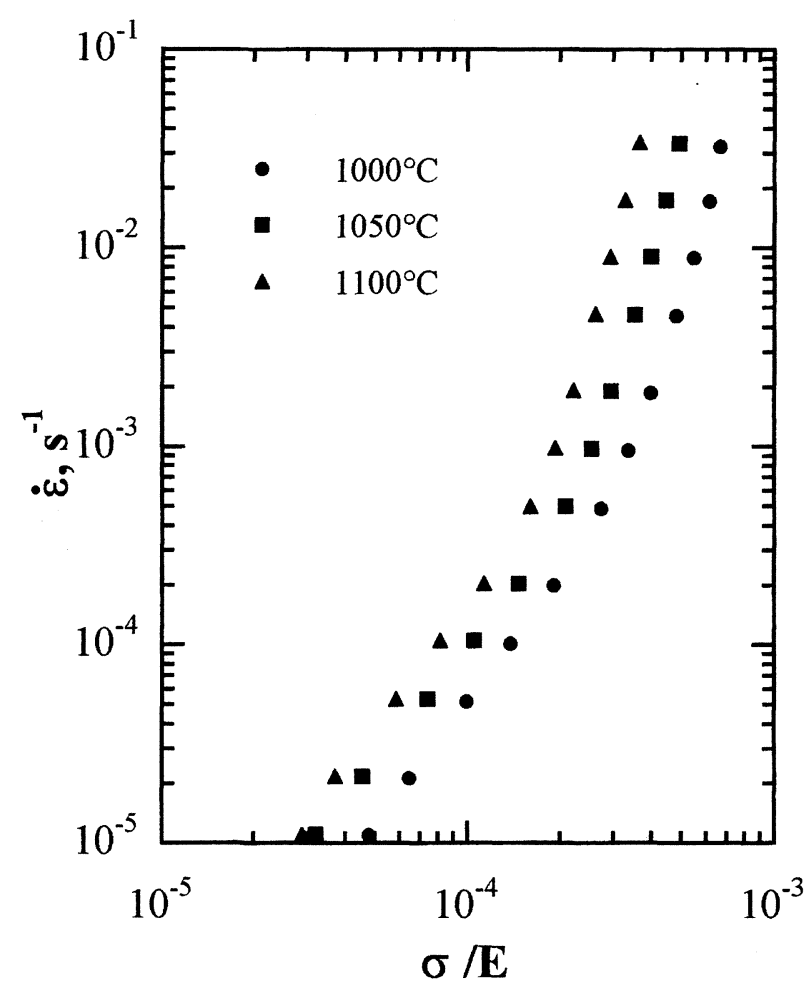

FIG. 3.- Velocidad de deformación en función de la tensión de fluencia corregida por el módulo de Young.

FIG. 3.- The strain rate as a function of Young's modulus compensated stress.

En la tabla I se presentan los resultados de los ensayos de tracción realizados a velocidad de deformación constante. Aunque se aprecia un comportamiento superplástico a ambas velocidades de deformación, se observa cómo un menor valor de $n$ va asociado a un mayor alargamiento a rotura.

\subsection{Evolución microestructural}

Con objeto de determinar los mecanismos que controlan la deformación plástica, se ha estudiado

TABLA I.- Variación del alargamiento a rotura con la temperatura y la velocidad de deformación del ensayo

TABLE I.- Variation of elongation to failure with temperature and testing strain rate

\begin{tabular}{|c|c|c|}
\hline Temperatura & $\dot{\varepsilon}, \mathrm{s}^{-1}$ & Alargamiento, \% \\
\hline $1.000^{\circ} \mathrm{C}$ & $10^{-4}$ & 250 \\
& $10^{-3}$ & 175 \\
$1.050^{\circ} \mathrm{C}$ & $10^{-4}$ & 300 \\
& $10^{-3}$ & 175 \\
$1.100{ }^{\circ} \mathrm{C}$ & $10^{-4}$ & 250 \\
& $10^{-3}$ & 170 \\
\hline
\end{tabular}

la microestructura presente en la zona de mordaza y en una región próxima a la superficie de fractura, en una probeta ensayada a rotura a $1.050{ }^{\circ} \mathrm{C}$ a una velocidad de deformación de $10^{-4} \mathrm{~s}^{-1}$. A medida que aumenta el grado de deformación plástica, las islas de granos austeníticos presentes en la figura $4 a$ van desapareciendo progresivamente $\mathrm{y}$, como muestra la figura $4 b$, desaparecen tras un $300 \%$ de deformación, alcanzándose una microestructura de granos aislados de austenita.

\section{DISCUSIÓN}

Para comprender el comportamiento mecánico del acero dúplex estudiado es necesario considerar la evolución de la microestructura a lo largo de la deformación del mismo. Los estudios metalográficos realizados muestran que, para conseguir
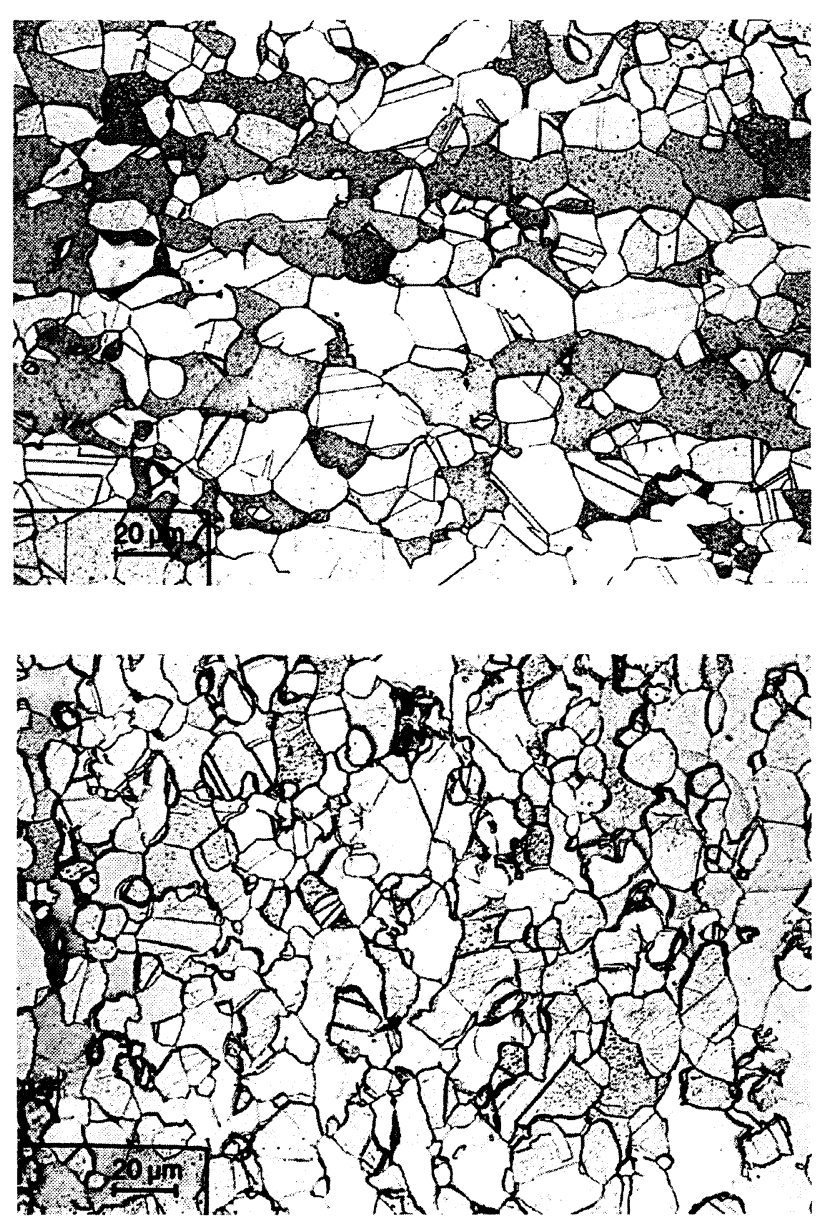

FIG. 4.- Microestructura después de un ensayo de tracción a $1.050{ }^{\circ} \mathrm{C}$ a $10^{-4} \mathrm{~s}^{-1}$. a) en la zona de mordaza y $b$ ) en una región próxima a la superficie de fractura.

FIG. 4.- Microstructure after tensile testing at $1,050^{\circ} \mathrm{C}$ at $10^{-4} \mathrm{~s}^{-1}$. a) in the grip region and b) in a region next to the fracture surface. 
un comportamiento superplástico del material, resulta necesario desarrollar en el mismo una microestructura de grano fino antes de ser ensayado. Para ello, se sometió al material a un proceso de laminación a temperatura ambiente. De este modo, antes de comenzar el ensayo de tracción, durante el tiempo de calentamiento para alcanzar la temperatura de ensayo, se consiguió la recristalización del material.

Esta microestructura de grano fino da lugar a un alargamiento a rotura $\geq 250 \%$ cuando se ensaya el material a temperaturas por encima de $1.000{ }^{\circ} \mathrm{C}$ a una velocidad de deformación de $10^{-4} \mathrm{~s}^{-1}$. Para esta velocidad de deformación, se encuentra, además, un valor del exponente de la tensión próximo a 2 , de modo que el mecanismo de deformación puede asociarse a un deslizamiento de fronteras de grano controlado por la autodifusión del hierro a lo largo de las fronteras de grano. Como consecuencia de este mecanismo de deformación, se produce una migración de los granos durante la deformación superplástica que da lugar a la desaparición de las colonias de granos austeníticos presentes en la cabeza de la probeta (Fig. 4).

A medida que aumenta la velocidad de deformación, comienza a ser importante la contribución, durante la deformación, de un mecanismo de deformación por movimiento de dislocaciones, lo cual explica tanto el aumento del valor del exponente de la tensión como la disminución del alargamiento a rotura. A una velocidad de deformación de $10^{-3} \mathrm{~s}^{-1}$ se obtiene un alargamiento a rotura del $170 \%$, que indica que a esta velocidad todavía es importante la contribución del mecanismo del deslizamiento de fronteras de granos. Sin embargo, a velocidades superiores, el mecanismo de deformación por movimiento de dislocaciones se hace dominante, alcanzándose un nuevo régimen de deformación caracterizado por un exponente de la tensión igual a 5 .

\section{CONCLUSIONES}

- El material de partida presenta una microestructura gruesa consistente en islas de austenita alar- gadas en la dirección de laminación, incluidas en una matriz más o menos continua de ferrita. Esta microestructura de partida se transforma en una microestructura con granos finos por recristalización del material durante el calentamiento a la temperatura del ensayo de tracción.

- Una microestructura de grano fino da lugar a un alargamiento a rotura superior al $250 \%$ cuando se ensaya el material a una velocidad de deformación de $10^{-4} \mathrm{~s}^{-1}$. Para esta velocidad de deformación, se encuentra, además, un valor del exponente de la tensión próximo a 2 , de modo que el mecanismo de deformación puede asociarse a un deslizamiento de fronteras de grano.

- A velocidades de deformación elevadas, el mecanismo de deformación por movimiento de dislocaciones se hace dominante, alcanzándose un nuevo régimen de deformación caracterizado por un exponente de la tensión igual a 5.

\section{Agradecimiento}

Los autores agradecen la financiación de este trabajo por la CICYT (Proyecto MAT 97/0700).

\section{REFERENCIAS}

(1) Gibson, R.G., Hayden, H.W. y Brophy, J.H. Trans. ASM, 61, 1968: 85-93

(2) HAYden, H.W. y FloReEn, S. Trans. ASM, 61, 1968: 474488.

(3) Floreen, S. y Hayden, H.W. Trans. ASM, 61, 1968: 489499.

(4) Forch, K., Gillessen, C., von Hagen, I. y Werling, W. Stahl und Eisen, 112, 1992: 53-62.

(5) Morley, J.I. y KirkBy, H.W. J. Iron Steel Inst., 172, 1952: 129-142.

(6) Maehara, Y., Ohmori, Y. y Kunitake, T. Met. Technol., 10, 1983: 296-303.

(7) Maehara, Y. y Langdon, T.G. Mater. Sci. Eng, A128, 1990: 1-13.

(8) Kobayashi, M. y Miyagawa, M. Trans. ISIJ, 27, 1987: 685-695. 